\title{
An Interesting Case of Hodgkin's Lymphoma Presenting as Salivary Gland Tumor
}

\author{
Tharun Ganapathy Chitrambalam¹, Sidhu Sekhar ${ }^{2}$, Preetham Anguraj ${ }^{3}$, Koshy M Panicker ${ }^{4}$
}

\begin{abstract}
A lump in the neck is a common problem presenting to clinicians. In most individuals, the new lump in the neck is noticed casually by the patient himself or by others. This lump without any associated symptoms may be the only indicator of underlying malignancy in the adult population. Here, we report one such case of a vague neck lump turning out to be lymphocyte-rich classical Hodgkin's lymphoma (HL). Lymphocyte-rich classical Hodgkin's lymphoma ( $\mathrm{LRCHL}$ ) is considered a rare subtype of $\mathrm{HL}$ having a relatively good prognosis.

Keywords: Hodgkin's lymphoma, Neck mass, Non-Hodgkin's lymphoma, Submandibular tumor.

International Journal of Head and Neck Surgery (2021): 10.5005/jp-journals-10001-1421
\end{abstract}

\section{INTRODUCTION}

A neck swelling has to be evaluated thoroughly to achieve a proper diagnosis. The proper way to approach any neck swelling begins with a good history taking and a complete clinical examination. ${ }^{1,2}$ Based on the location of the swelling and findings in the clinical examination, the possibilities can be narrowed down and the next step for the diagnosis can be determined. Common benign neck lumps include lipomas, thyroid nodules, tuberculous, infective, and inflammatory lymphadenitis. Congenital neck lumps include thyroglossal cyst, branchial cyst, dermoid cyst, and vascular anomalies. Malignant neck lumps include metastatic nodes, Hodgkin's lymphoma (HL), thyroid carcinomas, etc. ${ }^{3}$ Lymphomas are the third most common malignancy in the world and it constitutes around $3 \%$ of all malignant tumors. ${ }^{4,5}$ Hodgkin's lymphoma accounts for $10 \%$ of all lymphomas and is the most common type of lymphoma. The most common site of presentation of $\mathrm{HL}$ is mediastinum and neck followed by splenic, axillary, abdominal, hilar, or inguinofemoral. ${ }^{6}$ The most common type of presentation of $\mathrm{HL}$ is a painless progressive enlargement of the lymph nodes. The lymph nodes are usually smooth, firm, usually discrete, nontender with typical Indian rubber consistency. But in this case, it was palpable as a single swelling with irregular borders.

\section{Case Description}

A 41-year-old healthy male patient with no comorbidities presented to the surgical outpatient department with complaints of swelling in the left side of the neck which had increased in size over a period of 3 weeks. He was managed with a course of oral antibiotics by a primary care physician before reaching us. Owing to the continuous increase in size despite the antibiotic therapy he was referred to our tertiary hospital for further management. A history of mild pain over the swelling on deglutition was present. The patient had no history of night sweats, cough with expectoration, breathing difficulty, voice change, loss of weight, or any other constitutional symptoms. No history of any previous hospitalization and had no previous surgeries. On clinical examination, an ill-defined swelling in the neck which was approximately $5 \times 4 \mathrm{~cm}$ was palpable in the left side of the neck occupying the submandibular triangle. The skin over the swelling was normal. The consistency of the swelling was
${ }^{1}$ Department of General Surgery, Minimal Access Surgery, SRM Medical College and Research Institute, Chennai, Tamil Nadu, India

${ }^{2-4}$ Department of General Surgery, SRM Medical College and Research Institute, Chennai, Tamil Nadu, India

Corresponding Author: Tharun Ganapathy Chitrambalam, Department of General Surgery, Minimal Access Surgery, SRM Medical College and Research Institute, Chennai, Tamil Nadu, India, Phone: +91 9840044437, e-mail: drtarunchitrambalam@gmail.com

How to cite this article: Chitrambalam TG, Sekhar S, Anguraj P, et al. An Interesting Case of Hodgkin's Lymphoma Presenting as Salivary Gland Tumor. Int J Head Neck Surg 2021;12(2):89-91.

Source of support: Nil

Conflict of interest: None

firm. The swelling was non-fluctuant, nontender, and partly fixed to the underlying tissues. On palpating bimanually, the buccal mucosa was mildly projecting corresponding to the swelling, although no tenderness or discharge from the salivary ducts was noted. The floor of the oral cavity was soft without any abnormalities.

\section{INVESTIGATIONS}

Chest X-ray and all blood parameters were within normal limits. Complete tuberculosis (TB) workup was done and turned out to be negative. Ultrasonogram of the neck showed a $6 \times 4 \mathrm{~cm}$ complex cyst with solid components in the left submandibular region with increased vascularity and associated cervical lymphadenopathythe high possibility of submandibular tumor. Fine needle aspiration cytology (FNAC) was done twice and both reports showed an inconclusive study.

\section{Treatment}

On a provisional diagnosis of the submandibular tumor, neck exploration was done under general anesthesia. To our surprise, intraoperatively multiple swellings were noted in the submandibular region on the left side, the largest cystic swelling measuring $5 \times 3 \mathrm{~cm}$ with solid components (Fig. 1) and another $2 \times$ $2 \mathrm{~cm}$ solid swelling noted in the submandibular region extending into the posterior triangle of the neck which was adherent to the 


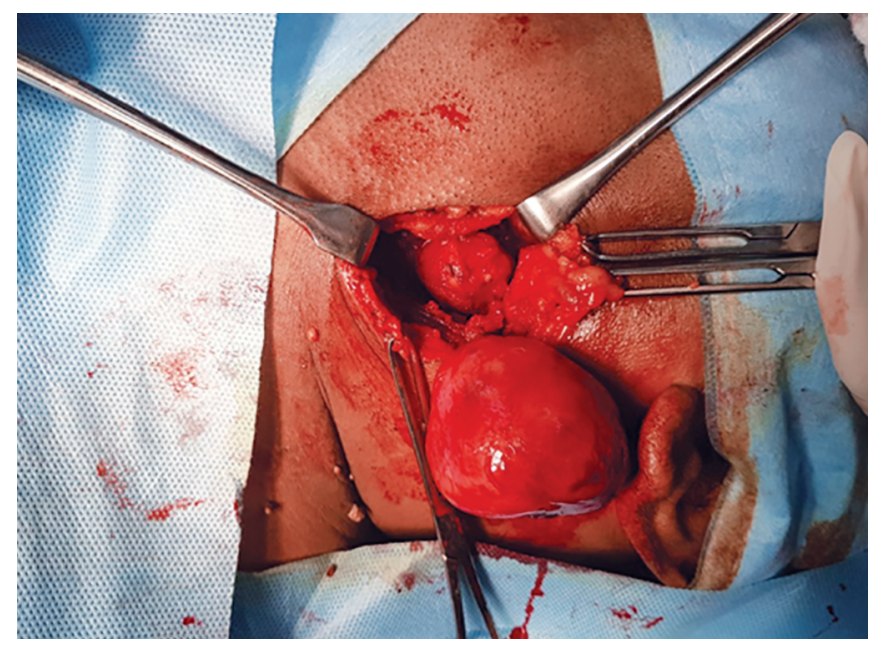

Fig. 1: Largest cystic swelling measuring $5 \times 3 \mathrm{~cm}$ with solid components and another $2 \times 2 \mathrm{~cm}$ solid swelling noted in the left submandibular region

surrounding muscles. Complete excision of the mass was done. The postoperative period was uneventful and the patient was discharged on postoperative day 2 .

\section{Pathological Discussion}

Histopathological sections studied showed lymph nodes with a pleomorphic population of lymphocytes, plasma cells, and histiocytes. A good number of histiocytic giant cells with binucleated Reed-Sternberg cells and occasional popcorn cells were noted in the study suggestive of classical HL-lymphocyte rich as in Figure 2.

\section{Follow-UP}

Positron emission tomography (PET) scan was subsequently done, which showed generalized lymphadenopathy with high uptake in all the nodes. The patient was started on adjuvant chemotherapy and completed a full course of treatment. The patient remained asymptomatic and was followed up for a period of 2 years. Repeat PET scan after 2 years showed no lymphadenopathy and the patient was disease-free.

\section{Discussion}

Lymphomas are common malignant tumors of the head and neck. Neck lymphomas usually present with constitutional symptoms like fever, fatigue, and malaise and are commonly seen occupying the posterior triangle of the neck ${ }^{7}$. Hodgkin's lymphoma is a solid tumor that arises from $B$ lymphocytes and is characterized by the presence of Reed-Sternberg cells. It was first described in $1832 .{ }^{8,9}$ The cervical lymphadenopathy in $\mathrm{HL}$ presents as a painless swelling that has a rubbery texture when compared with that of the secondaries in the neck. As the disease progresses, there will be diffuse lymphadenopathy and also splenomegaly. ${ }^{10}$ Differential diagnosis of subacute development of neck lumps includes common benign neck lumps like thyroid nodules, tuberculous, infective, and inflammatory lymphadenitis to malignant neck lumps like metastatic nodes, $\mathrm{HL}$, thyroid carcinomas, etc. ${ }^{3}$ Rare neck masses include (A) human papillomavirus-related squamous cell carcinoma

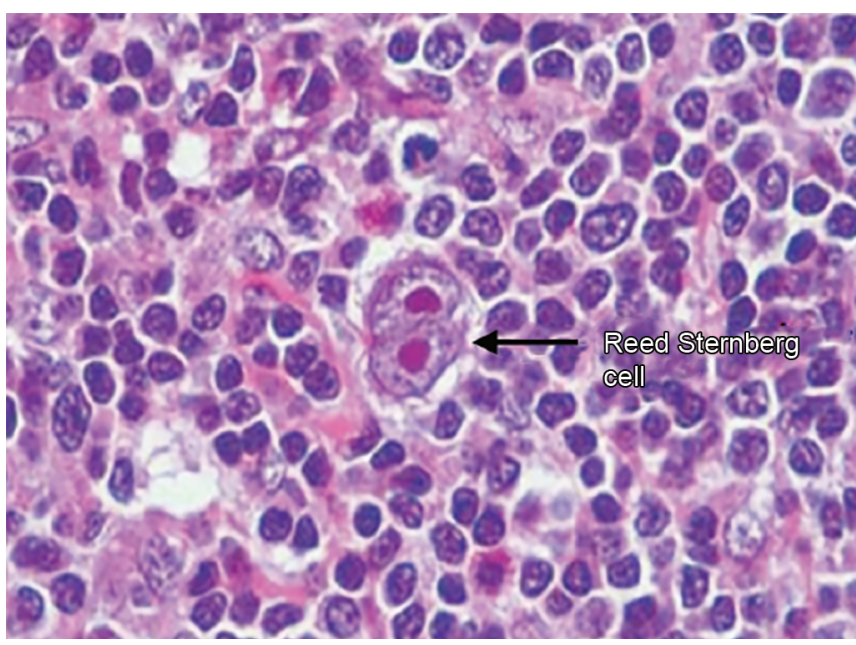

Fig. 2: Histopathological slide showing the presence of Reed-Sternberg cell confirming Hodgkin's lymphoma with subtype LRCHL (lymphocyterich classical Hodgkin's lymphoma)

which can present with rapidly enlarging lateral lymph nodes masses, asymmetry in palatine or lingual tonsils, dysphagia, change of voice, or pharyngeal bleeding; (B) metastatic cancer which can present with matted, firm, or fixed lymph nodes; (C) non-HL which present as painless, rapidly growing lymph node mass which is mobile and rubbery in consistency and can also involve the tonsillar ring in the pharynx; (D) chronic sialadenitis of the submandibular salivary gland and (E) idiopathic conditions like Castleman disease, Rosai-Dorfman disease, Kimura disease, and Kikuchi disease (histiocytic necrotizing lymphadenitis). All of the above can present as asymptomatic neck masses in the submandibular region and mimic HL. ${ }^{11}$ FNAC has a very limited role but is helpful to rule out tuberculous swellings or secondaries in the neck. Excision biopsy is generally preferred and the entire lymph node is removed to retain its architecture. Other markers of immunohistochemistry like CD15, CD30, and CD45 are useful in confirming the diagnosis of lymphoma. PET CT is usually done to identify extranodal involvement. The treatment strategy, however, mainly revolves around radiotherapy and chemotherapy. According to Ann-Arbor clinical staging, stages I and II are treated with radiotherapy while III and IV are treated with systemic chemotherapy.

\section{Conclusion}

Hodgkin's and non-HL can present as isolated neck lumps without any other manifestations in about $4.54 \%$ of all lymphoma patients. Although only a neck mass is clinically appreciated at presentation, it can be a manifestation of an advanced stage disease as seen in this case. This case serves to emphasize the importance of a thorough evaluation of an asymptomatic neck lump, especially in the adult population to diagnose rare cancers and improve outcomes.

\section{References}

1. Alvi A, Johnson JT. The neck mass. Postgrad Med 1995;97(5):87-97. DOI: 10.1080/00325481.1995.11945993.

2. Weymuller Jr EA. Evaluation of neck masses. J Fam Pract 1980;11:1099.

3. Dickson PV, Davidoff AM. Malignant neoplasms of the head and neck. Semin Pediatr Surg 2006;15:92-98. 
4. Lu P. Staging and classification of lymphoma. Semin Nucl Med 2005;35:160-164.

5. Inchingolo F, Tatullo M, Abenavoli FM, et al. Non-Hodgkin lymphoma affecting the tongue: unusual intra-oral location. Head Neck Oncol 2011;3(1):1-5. DOI: 10.1186/1758-3284-3-1.

6. Mauch PM, Kalish LA, Kadin M, et al. Patterns of presentation of Hodgkin disease. Implications for etiology and pathogenesis. Cancer 1993;71(6):2062-2071.

7. Daskalopoulou D, Papanastasiou C, Markidou S, et al. The diagnostic value of fine needle aspiration cytology in Waldeyer's ring lymphomas. Eur J Cancer [Oral Oncol] 2001;37:36.
8. Buglio D, Younes A. Histone deacetylase inhibitors in Hodgkin lymphoma. Invest New Drugs 2010;28(suppl 1):S21-S27. DOI: 10.1007/ s10637-010-9588.

9. Hurtado-Cordovi JM, Verma V, Gotlieb V, et al. Lymphocyte rich Hodgkin's lymphoma presented with warm hemolytic anemia: a case report and literature review. Case Rep Hematol 2011;2011:385408. DOI: 10.1155/2011/385408.

10. Abramson JS, Digumarthy S, Ferry JA. Case records of the Massachusetts General Hospital. N Engl J Med 2009;361:900-911.

11. Haynes J, Arnold KR, Aguirre-Oskins C, et al. Evaluation of neck masses in adults. Am Fam Physician 2015;91(10):698-706. 